\title{
A case of convergence spasms - do not be caught off-guard
}

\author{
L Smit, MB ChB, MMed (Neurol) \\ Department of Neurology, Faculty of Health Sciences, University of the Free State, Bloemfontein, South Africa
}

Corresponding author: L Smit (smitl@ufs.ac.za)

\begin{abstract}
A hypertensive patient presented with complaints of headache and fluctuating double vision and deterioration in vision from 2010. She displayed symptoms associated with convergence spasms, which could be confused with sixth cranial nerve palsies. A few pointers are given to prevent clinicians from being caught off-guard when encountering this clinical condition.
\end{abstract}

S Afr Med J 2016;106(12):1188-1189. DOI:10.7196/SAMJ.2016.v106i12.11417

\section{Case report}

A 42-year-old woman presented in 2014 with gradual onset of frontotemporal headaches associated with nausea and vomiting since 2010, following the birth of her youngest child. She also had complaints of double vision and deterioration of vision since 2010. The diplopia worsened on near fixation of objects. Occasional episodes of dizziness occurred after taking antihypertensive medication.

She was known to have hypertension, treated with four agents, and had evidence of grade 4 hypertensive retinopathy. She had attempted suicide previously with antihypertensive medication. On examination she was alert, responsive, orientated to time and place, with no signs of raised intracranial pressure and meningeal irritation. No vomiting occurred in the ward. Pupils were equal and reactive to light. Visual acuity in both eyes was 20/40 on the Rosenbaum pocket vision screener.

As demonstrated in Fig. 1, the patient had dysconjugate gaze in the primary position with the eyes adducted (turned toward the nose bridge). Fluctuating esotropia was present. When the object for fixation was held closer than $30 \mathrm{~cm}$ to examine the eye movements, the patient developed convergence and miosis was noted to occur synergistically. When eye movements were tested separately, they were normal with full range of pursuit movements and saccades. Bilateral contraction of the orbicularis oculi muscles was also noted. No other cranial nerve abnormalities or long tract signs were observed. The diagnosis of convergence spasm (spasm of the near reflex) was made.

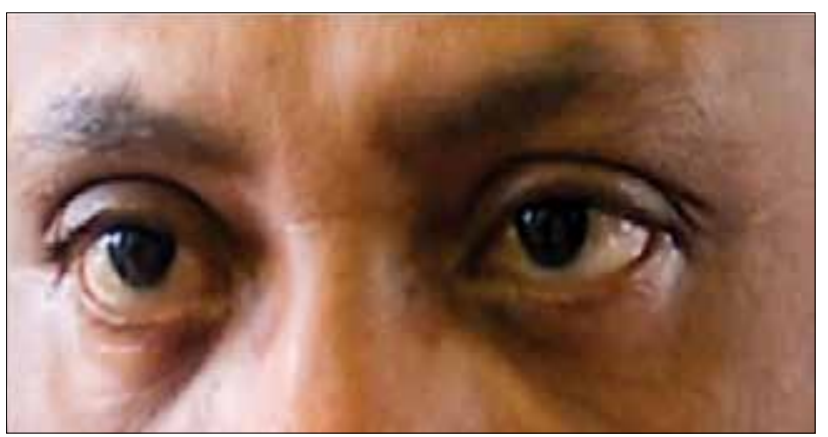

Fig. 1. Patient showing dysconjugate gaze in the primary position with the eyes adducted.
The results of routine blood tests were normal. Urea and electrolytes, liver function, vitamin $B_{12}$, full blood count and erythrocyte sedimentation rate were normal, and tests for Treponema pallidum and HIV were negative. Computed tomography (CT) of the brain was performed, which was essentially normal and did not disclose intraor extra-axial space-occupying lesions. Asymmetry of the lateral ventricles was noted but was considered insignificant.

During her stay in the ward, an incident occurred when her husband came to visit her. He was verbally and physically abusive towards her. She refused support from the social worker. When it was explained to her that her eye movements were stress-related, she accepted it as the cause of her problems. She was reassured that the movements should clear up as soon as the stress was reduced. On follow-up every morning in the ward, the convergence spasms became less noticeable. She was discharged with normal eye movements after a 2-week stay in hospital.

This patient had presented in 2014 to physicians who were less experienced in neuro-ophthalmology. She had been wrongly diagnosed as suffering from bilateral sixth cranial nerve palsies as a result of raised intracranial pressure.

\section{Discussion}

Spasm of the near reflex is a disorder characterised by intermittent episodes of convergence, miosis and accommodation. It may mimic bilateral and sometimes unilateral nervus abducens paresis. ${ }^{[1]}$ The patient complains of double vision or blurred vision. Prominent miosis on convergence is the clue in differentiating convergence spasm from true organic causes of convergence phenomena. ${ }^{[2]}$ Spasm of the near reflex as described above may rarely occur in patients with organic disorders, but is more commonly psychogenic. ${ }^{[3,4]}$

Spasm of the near reflex is one cause for esotropia. Organic disorders causing esotropia include conditions such as abducens palsy, tonic convergence spasm (part of dorsal midbrain syndrome), pons lesions in multiple sclerosis, ${ }^{[5]}$ myasthenia gravis and Wernicke's encephalopathy. ${ }^{[1]}$ Patients with functional spasm of the near reflex have associated somatic complaints and behavioural abnormalities. Blepharoclonus (frequent blinking), poor co-operation in other motor tasks and tunnel vision may occur. The patient has a full range of eye movement with pursuit of own hand and with one eye covered. Normal optokinetic nystagmus is observed. ${ }^{[1]}$ 
A few pointers that may be of value when examining a patient with convergence spasms include the following:

- Should eye signs be confusing or fluctuating, it is useful to cover one eye and examine eye movements independently.

- The fixation point should not be closer than $50 \mathrm{~cm}$ to the face of the patient when examining eye movements.

- Check for miosis on convergence when unexplained 'sixth cranial nerve' palsies occur. Presence of such indicates a physiologically normal near response.

- Look for contraction of the orbicularis oculi muscle if convergence spasm is suspected, in which case associated contraction of this muscle of the face during eye movement examination usually also occurs.

- This condition in isolation seldom needs special investigations.
- Psychological support is usually indicated and may assist in spontaneous remission of the convergence spasms.

1. Lavin PJM. Neuro-ophthalmology: Ocular motor system. In: Daroff RB, Fenichel GM, Jankovic J, Mazziotta JC, eds. Bradleys' Neurology in Clinical Practice. Vol. 1. Principles of Diagnosis and Management. 6th ed. Philadelphia: Elsevier Saunders, 2012:87-633.

2. Anagnostou E, Katsika P, Kemanetzoglou E, Vassilopoulou S, Spengos K. The abduction deficit of functional convergence spasm. J Neurol Sci 2016;363:27-28. http://dx.doi.org/10.1016/j.jns.2016.02.027

3. Fekete R, Baizabal-Carvallo JF, Ha AD, Davidson A, Jankovic J. Convergence spasm in conversion disorders: Prevalence in psychogenic and other movement disorders compared with controls. I Neurol Neurosurg Psychiatry 2012;83(2):202-204. http://dx.doi.org/10.1136/jnnp-2011-300733

4. Ghosh A, Padhy SK, Gupta G, Goyal MK. Functional convergence spasm. Indian J Psychol Med 2014;36(3):332-334.

5. Anliaçik S, Uca AU, Kozak HH, Akpinar Z. A very rare paroxysmal symptom in multiple sclerosis: Convergence spasm. Am J Emerg Med 2016;34(1):117.e5-117.e6. http://dx.doi.org/10.1016/j. ajem. 2015.05 .025

Accepted 22 August 2016. 